\title{
One-year follow-up—case report of secondary tension pneumothorax in a COVID-19 pneumonia patient
}

\author{
Felix Endres ${ }^{1} \cdot$ Judith Eva Spiro ${ }^{2} \cdot$ Toki Anna Bolt $^{3} \cdot$ Amanda Tufman $^{3} \cdot$ Ben Ockert $^{1} \cdot$ Tobias Helfen $^{1} \cdot$ Fabian Gilbert $^{1}$. \\ Boris M. Holzapfel ${ }^{1}$. Wolfgang Böcker ${ }^{1} \cdot$ Georg Siebenbürger ${ }^{1}[$
}

Received: 4 August 2021 / Accepted: 24 September 2021 / Published online: 8 October 2021

(c) The Author(s) 2021

\begin{abstract}
Purpose The Coronavirus Disease 2019 (COVID-19) may result not only in acute symptoms such as severe pneumonia, but also in persisting symptoms after months. Here we present a 1 year follow-up of a patient with a secondary tension pneumothorax due to COVID-19 pneumonia.

Case presentation In May 2020, a 47-year-old male was admitted to the emergency department with fever, dry cough, and sore throat as well as acute chest pain and shortness of breath. Sputum testing (polymerase chain reaction, PCR) and computed tomography (CT) confirmed infection with the severe acute respiratory syndrome coronavirus type 2 (SARS-CoV-2). Eleven days after discharge, the patient returned to the emergency department with pronounced dyspnoea after coughing. CT showed a right-sided tension pneumothorax, which was relieved by a chest drain (Buelau) via mini open thoracotomy. For a period of 3 months following resolution of the pneumothorax the patient complained of fatigue with mild joint pain and dyspnoea. After 1 year, the patient did not suffer from any persisting symptoms. The pulmonary function and blood parameters were normal, with the exception of slightly increased levels of D-Dimer. The CT scan revealed only discrete ground glass opacities (GGO) and subpleural linear opacities.

Conclusion Tension pneumothorax is a rare, severe complication of a SARS-CoV-2 infection but may resolve after treatment without negative long-term sequelae.
\end{abstract}

Level of evidence $\mathrm{V}$.

Keywords COVID-19 $\cdot$ Pneumothorax $\cdot$ Pneumonia $\cdot$ Multi-detector computed tomography $\cdot$ Viral infections

\section{Background}

The first infections with the severe acute respiratory syndrome coronavirus type 2 (SARS-CoV-2) were detected in humans in late 2019 [1]. While much is known about the acute symptoms of the Coronavirus Disease 2019

Georg Siebenbürger

georg.siebenbuerger@med.uni-muenchen.de

1 Department of Orthopaedics and Trauma Surgery, Musculoskeletal University Center Munich (MUM), University Hospital, LMU Munich, Ziemssenstraße 5, 80336 Munich, Germany

2 Department of Radiology, University Hospital, LMU Munich, Munich, Germany

3 Department of Medicine V, University Hospital, Member of the German Center for Lung Research (DZL), LMU Munich, Munich, Germany
(COVID-19), long-term health effects of surviving patients remain unknown. Patients' most common symptoms are fatigue, cognitive symptoms, dyspnoea, joint pain, and chest pain $[2,3]$.

In June 2020, we reported a case of a secondary tension pneumothorax as a complication of COVID-19 pneumonia [4]. Reports of pneumothorax as a complication of COVID19 are rare and the reported pneumothoraces are often ventilator-induced $[5,6]$. To our knowledge, a 1 year follow-up of a secondary tension pneumothorax due to COVID-19 infection has not been published so far.

\section{Case report}

A 47-year-old male was admitted to our emergency department with dry cough, shortness of breath, and stenocardia. The symptoms had started 14 days before primary 
admission. Polymerase chain reaction (PCR) tests of nasal and pharyngeal swabs and sputum were positive for SARSCoV-2-RNA N-gene 1, and negative for respiratory syncytial virus (RSV), Influenza-A and Influenza-B. Antibody titres (Anti-SARS-CoV-2-IgG and Anti-SARS-CoV-2-IgA) were positive and SARS-CoV-2-RNA N-gene 1 (PCR) was negative 5 days after primary admission.

The patient had no previously known pulmonary or thoracic diseases. Due to a traumatic motorcycle accident, the patient had undergone splenectomy years before. The patient was under treatment with Dovato ${ }^{\circledR} 50 / 300 \mathrm{mg}\left(\mathrm{GSK}^{\circledR}\right.$, Dolutegravir/Lamivudine) human immunodeficiency virus (HIV) infection, HIV-1-RNA testing (PCR) showed a level of $<40$ copies $/ \mathrm{mL}$. The patient was treated for COVID-19 pneumonia with supportive measures and discharged with lowering CRP levels as well as normalized leucocytes and interleukine- 6 levels after 7 days.

Four days later, 11 days after his initial presentation, the patient was admitted to the emergency department with pronounced dyspnoea after coughing. The clinical and radiographic examination revealed a right-sided tension pneumothorax. To relieve the tension pneumothorax, a small caliber chest tube $(20$ Charrière $=$ French, Buelau $)$ was inserted through a mini open thoracotomy in the 5th right intercostal space. After an almost complete regression of the pneumothorax the chest tube was removed after 8 days and the patient was discharged after 9 days (20 days after primary admission) in stable condition.

For a period of 3 months after discharge, the patient complained of fatigue with mild joint pain and dyspnoea. After 3 months, the patient reported no residuals of the infection or the tension pneumothorax. During the course of a year, he had regular check-ups including a pulmonary function test, blood tests, X-rays, and computed tomography (CT) scans that are outlined in detail in the following.
The clinical examination 12 months after first admission revealed no persisting symptoms. The surgical wound fully healed without irritation. The soft tissue showed a satisfying aesthetic result, the scar being clean and dry.

After 12 months, the 36-item short-form health survey (SF-36) revealed no limitations in physical functioning (average 100), no role limitations due to physical health (average 100) or due to emotional problems (average 100), no signs of fatigue (average 90), no loss of emotional wellbeing (average 96) or social functioning (average 100), no indication of pain (average 100) and a good state of general health (average 100) [7].

The Quality of live survey (EQ-5D-5L) also showed no limitation in mobility, self-care, usual activities, pain, and anxiety/depression.

Unfortunately, patient-reported quality of life was not assessed at the time of acute diagnosis.

Figure 1 presents the timeline of the events.

\section{Chest X-rays and CT scans}

Eight days after second admission, thoracic X-rays showed right-sided soft tissue emphysema and bipulmonary opacities due to COVID-19 pneumonia. The pneumothorax was no longer visible on radiographs. CT, however, showed residuals of the pneumothorax, as well as soft tissue emphysema and mild pneumomediastinum. Furthermore, bipulmonary signs of COVID-19 pneumonia (ground glass opacities (GGO) and consolidations with peripheral distribution) were depicted on CT images at this time point. Thoracic $\mathrm{X}$-ray performed 1 month after second discharge from the hospital showed regressive pulmonary opacities due to COVID-19 pneumonia. Further chest X-rays performed 8, 9, and 10 months after second discharge were all normal.

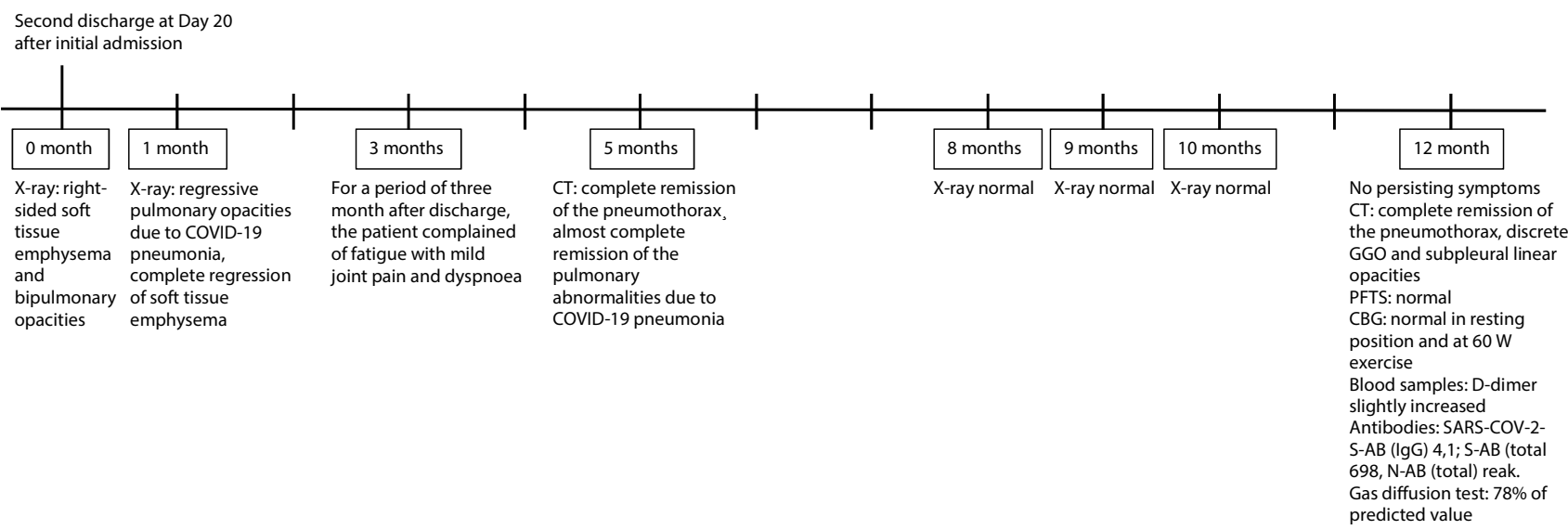

Fig. 1 Timeline of the patient history from second discharge to 1 year follow-up. COVID-19 Coronavirus Disease 2019, CT computed tomography, GGO ground glass opacities, SARS-CoV-2 severe acute respiratory syndrome coronavirus $2, A B$ antibodies, PFTS pulmonary function tests, $C B G$ capillary blood gas 
CT scans acquired 5 and 12 months after second discharge showed complete remission of the pneumothorax and almost complete remission of the pulmonary abnormalities due to COVID-19 pneumonia. One year after second discharge only discrete GGO and subpleural linear opacities, presumably corresponding to mild post-inflammatory fibrotic changes, remained detectable on CT images (Fig. 2) [8].

\section{Clinical course}

Pulmonary function tests after 12 months showed a forced vital capacity (FVC) of $103 \%$ of predicted value $(6360 \mathrm{~mL})$, and a forced expiratory volume in one second (FEV 1) of $109 \%$ of predicted value $(5240 \mathrm{~mL})$. Capillary blood gas

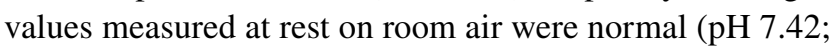
pCO2 $37.5 \mathrm{mmHg}$; pO2 $80.7 \mathrm{mmHg}$ and $\mathrm{SO} 297 \%$ ) and there was a normal physiological increase in $\mathrm{pO} 2$ with light exercise $(60 \mathrm{~W})$ resulting in $\mathrm{pH} 7.41$; pO2 $86.3 \mathrm{mmHg}$, pCO2 $38.3 \mathrm{mmHg}, \mathrm{SO} 297 \%$. The gas diffusion test revealed a minimally decreased diffusion capacity of $78 \%$ of predicted value $[12.36 \mathrm{mmol} /(\min * \mathrm{kPa})]$.

\section{Blood samples}

Initially, in March 2020, laboratory parameters showed increased levels of CRP $(10.2 \mathrm{mg} / \mathrm{d})$, lactate dehydrogenase (LDH) (406 U/L), leucocytes (12.4 G/L), D-dimers $(1.7 \mu \mathrm{g} /$ $\mathrm{mL})$ and interleukine- $6(122 \mathrm{pg} / \mathrm{mL})$.

The blood samples after 12 months revealed normal CRP $(0.4 \mathrm{mg} / \mathrm{dl}), \mathrm{LDH}(231 \mathrm{U} / \mathrm{L})$, leucocytes $8,49 \mathrm{G} / \mathrm{L}$ and Interleukine-6 (5.4 pg/ml). Neutrophil count (41\%) and lymphocyte count $(45 \%)$ were also normal. D-Dimer levels were still increased $(0.9 \mu \mathrm{g} / \mathrm{mL})$ but lowered compared to the D-dimer levels in March 2020.

Tests for SARS-CoV-2 N antibodies (total; Roche) were positive $(698 \mathrm{U} / \mathrm{ml})$, as were as SARS-CoV-2 $\mathrm{S}$ antibodies (IgG; Euroim.) (4:1 ratio).

One month before admission, at a regular check-up, CD4 count was 1408 cells/ $\mu \mathrm{l}$ (35\% of lymphocytes) and CD4/ CD8 ratio 0.9. Nadir CD4 count during the subsequent COVID-19 illness was 573 cells/ $\mu$ ( $23 \%$ of lymphocytes) and lowest CD4/CD8 ratio 0.472 days after hospitalization. Three months after discharge the CD4 count was 1445 cells/ $\mu \mathrm{l}$ (57\% of lymphocytes) and CD4/CD8 ratio 0.84 . One year after discharge the CD4 count was 1195 cells/ $\mu \mathrm{l}$ (45\% of lymphocytes) and CD4/CD8 ratio 0.91 .

\section{Discussion}

Since the beginning of the pandemic in late 2019 little is known about long-term sequelae of COVID-19. Carfi et al. reported that $87 \%$ of hospitalized COVID-19 patients $(n=143)$ continued to experience at least one persistent symptom 60 days after onset of the disease, and $44 \%$ had worsened quality of life [3]. In another study, Taboada et al. found that 6 months after hospitalization the functional status decreased in $47.5 \%$ of the patients. Fumagalli et al. examined the respiratory function at the time of clinical recovery and 6 weeks after discharge in 13 patients surviving COVID-19 pneumonia. Their findings suggest that COVID19 pneumonia may result in clinically relevant alterations in pulmonary function tests, with a mainly restrictive pattern [9]. In our case, pulmonary function tests after a year were normal without a sign of a restrictive pattern. Gas diffusion test revealed a decrease of the diffusion capacity of $78 \%$ of predicted value.

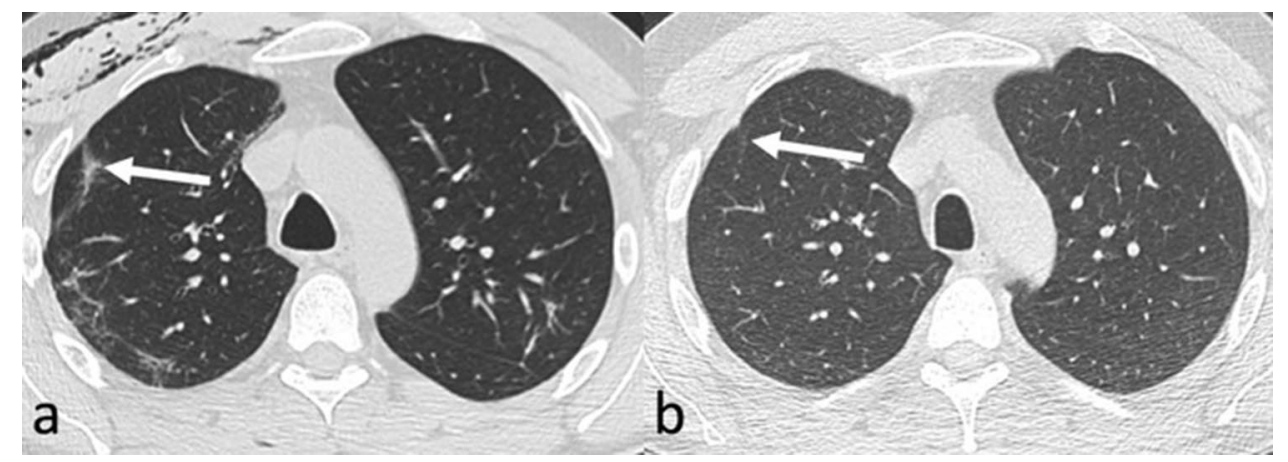

Fig. 2 Radiological follow-up after tension pneumothorax due to COVID-19 pneumonia. Thoracic computed tomography performed 1 day before second discharge from the hospital (a) shows subpleural linear and reticular consolidations as a sign of COVID-19 pneumonia (arrow). Furthermore, there is right-sided soft tissue emphysema due to the pneumothorax (the latter not shown). One year later (b), the pulmonary abnormalities due to COVID-19 pneumonia have almost completely resolved. Only very few, discrete subpleural linear consolidations and GGO (arrow), which presumably correspond to minor post-inflammatory fibrotic changes, are visible. COVID-19 coronavirus disease 2019, $C T$ computed tomography, $G G O$ ground-glass opacities 
Pneumothoraces in association with COVID-19 infections are still a rare complication. Spontaneous pneumothoraces were described in only $1 \%$ of cases, predominantly in male patients $(3.3: 1)$ [10,11]. Delayed pneumothoraces were reported in several cases $[12,13]$. While the exact process of the development of a delayed pneumothorax remains unknown, persistent chronic inflammatory changes and delayed alveolar damage due to coughing are discussed as a possible cause [14]. In our case, the patient was admitted to the hospital with pneumothorax after coughing.

Morin et al. presented an uncontrolled cohort study of 478 survivors of COVID-19 4 months after hospitalization. 244 patients $(51 \%)$ reported at least one new-onset symptom. The most common symptoms were fatigue in 134 of 431 (31\%), cognitive impairments in 86 of $416(21 \%)$, and dyspnoea in 78 of $478(16 \%)$ patients. CT lung scan abnormalities were reported in $63 \%$ of 171 patients assessed at an ambulatory visit, mainly subtle GGO. Fibrotic lesions were observed in $19 \%$ of these 171 patients [2]. These findings are in line with the clinical manifestations of our case. Here the patient showed relevant symptoms for the first 3 months after the infection and secondary tension pneumothorax. One year after hospitalization, the patient was asymptomatic with no loss in quality of life. The CT scan revealed only subtle GGO and subpleural linear opacities, presumably corresponding to mild post-inflammatory fibrotic changes.

Immunological dysfunction such as HIV infection is most likely a risk factor for disease severity $[15,16]$. However, this could not be confirmed in a German series of $33 \mathrm{HIV}$ infected patients with COVID-19 and a series of $26 \mathrm{HIV}-$ infected, COVID-19 diagnosed patients in northern Italy, where morbidity and mortality of COVID-19 were comparable to those reported in other cohorts of HIV-negative patients $[17,18]$. In our case, based on the blood samples and the medical history of the patient within the last year, the HIV infection showed no progression with stable CD4/ CD8 ratio. Furthermore, blood samples revealed normal levels for CRP, LDH, leucocytes, and Interleukine- 6 while the antibody titers were still relatively high.

Townsend et al. reported increased D-dimer levels up to 4 months in $25 \%$ of patients after SARS-CoV-2 infection. In our patient, D-dimer levels were still elevated 1 year after Covid-19 [19].

\section{Conclusion}

We present 1 year follow-up of a case of secondary tension pneumothorax as a complication of SARS-CoV-2 infection. After 1 year, the patient did not suffer from any persisting symptoms. The pulmonary function was normal as were the blood parameters except the D-Dimer levels, which remained slightly increased. The CT scan revealed only discrete GGO and subpleural linear opacities. The HIV infection showed no progression.

Acknowledgements We thank the patient for granting us permission to publish this information.

Author contributions All authors contributed to the study conception and design. FE, JES, TAB and GS performed material preparation, data collection and analysis. FE wrote the first draft of the manuscript and all other authors commented on previous versions of the manuscript. All authors read and approved the final manuscript.

Funding Open Access funding enabled and organized by Projekt DEAL. This manuscript was not funded.

Availability of data and material All available information is contained within the manuscript.

\section{Declarations}

Conflict of interest On behalf of all authors, the corresponding author states that there are no conflicts of interest.

Ethics approval Ethics approval was not obtained due to clinical standard treatment.

Consent to participate Written informed consent was obtained from the patient.

Consent for publication Written informed consent was obtained from the patient for publication of this case and any accompanying images. A copy of the written consent is available for review by the Editor of this journal.

Open Access This article is licensed under a Creative Commons Attribution 4.0 International License, which permits use, sharing, adaptation, distribution and reproduction in any medium or format, as long as you give appropriate credit to the original author(s) and the source, provide a link to the Creative Commons licence, and indicate if changes were made. The images or other third party material in this article are included in the article's Creative Commons licence, unless indicated otherwise in a credit line to the material. If material is not included in the article's Creative Commons licence and your intended use is not permitted by statutory regulation or exceeds the permitted use, you will need to obtain permission directly from the copyright holder. To view a copy of this licence, visit http://creativecommons.org/licenses/by/4.0/.

\section{References}

1. Zhu N, et al. A novel coronavirus from patients with pneumonia in China, 2019. N Engl J Med. 2020;382:727-33.

2. Group, T.W.C.f.t.C.S. Four-month clinical status of a cohort of patients after hospitalization for COVID-19. JAMA. 2021;325:1525-34.

3. Carfì A, et al. Persistent symptoms in patients after acute COVID19. JAMA. 2020;324:603-5.

4. Spiro JE, et al. Secondary tension pneumothorax in a COVID-19 pneumonia patient: a case report. Infection. 2020;48:941-4. 
5. Akdogan RE, et al. Pneumothorax in mechanically ventilated patients with COVID-19 infection. Case Rep Crit Care. 2021;2021:6657533.

6. Dennison J, et al. Case report: spontaneous pneumothorax in resolved, uncomplicated COVID-19 pneumonia-a literature review. Respir Med Case Rep. 2020;31:101291.

7. Ware JE Jr, Sherbourne CD. The MOS 36-item short-form health survey (SF-36). I. Conceptual framework and item selection. Med Care. 1992;30:473-83.

8. Wang F, Kream RM, Stefano GB. Long-term respiratory and neurological sequelae of COVID-19. Med Sci Monit. 2020;26:e928996.

9. Fumagalli A, et al. Pulmonary function in patients surviving to COVID-19 pneumonia. Infection. 2021;49:153-7.

10. Chen N, et al. Epidemiological and clinical characteristics of 99 cases of 2019 novel coronavirus pneumonia in Wuhan, China: a descriptive study. Lancet. 2020;395:507-13.

11. Martinelli AW et al. COVID-19 and pneumothorax: a multicentre retrospective case series. Eur Respir J. 2020;56(5):2002697. https://doi.org/10.1183/13993003.02697-2020. Accessed 19 Nov 2020; Print Nov 2020

12. Kasturi S, et al. Delayed recurrent spontaneous pneumothorax post-recovery from COVID-19 infection. Indian J Thorac
Cardiovasc Surg. 2021;37(5):1-3. https://doi.org/10.1007/s12055021-01145-w. Accessed 2 Feb 2021 (Online ahead of print).

13. Shah V, et al. Delayed recurrent spontaneous pneumothorax in a patient recovering from COVID-19 pneumonia. Korean J Anesthesiol. 2021;74:183-5.

14. Marzocchi, G., A. Vassallo, and F. Monteduro, Spontaneous pneumothorax as a delayed complication after recovery from COVID19. BMJ Case Rep, 2021. 14(5).

15. Wolff $\mathrm{D}$, et al. Risk factors for Covid-19 severity and fatality: a structured literature review. Infection. 2021;49:15-28.

16. Mirzaei H, et al. COVID-19 among people living with HIV: a systematic review. AIDS Behav. 2021;25:85-92.

17. Härter G, et al. COVID-19 in people living with human immunodeficiency virus: a case series of 33 patients. Infection. 2020;48:681-6.

18. Calza L, et al. COVID-19 in patients with HIV-1 infection: a single-centre experience in northern Italy. Infection. 2021;49:333-7.

19. Townsend L, et al. Prolonged elevation of D-dimer levels in convalescent COVID-19 patients is independent of the acute phase response. J Thromb Haemost. 2021;19:1064-70. 